\title{
Bending and Stretching of Soft Pores Enable Passive Control of Fluid Flows
}

\author{
Louf, Jean François; Knoblauch, Jan; Jensen, Kaare H.
}

Published in:

Physical Review Letters

Link to article, DOI:

10.1103/PhysRevLett.125.098101

Publication date:

2020

Document Version

Publisher's PDF, also known as Version of record

Link back to DTU Orbit

Citation (APA):

Louf, J. F., Knoblauch, J., \& Jensen, K. H. (2020). Bending and Stretching of Soft Pores Enable Passive Control of Fluid Flows. Physical Review Letters, 125(9), [098101]. https://doi.org/10.1103/PhysRevLett.125.098101

\section{General rights}

Copyright and moral rights for the publications made accessible in the public portal are retained by the authors and/or other copyright owners and it is a condition of accessing publications that users recognise and abide by the legal requirements associated with these rights.

- Users may download and print one copy of any publication from the public portal for the purpose of private study or research.

- You may not further distribute the material or use it for any profit-making activity or commercial gain

- You may freely distribute the URL identifying the publication in the public portal 


\title{
Bending and Stretching of Soft Pores Enable Passive Control of Fluid Flows
}

\author{
Jean-François Louf@, Jan Knoblauch, and Kaare H. Jensen ${ }^{*}$ \\ Department of Physics, Technical University of Denmark, DK-2800 Kgs, Lyngby, Denmark
}

(Received 9 August 2019; accepted 13 July 2020; published 25 August 2020)

\begin{abstract}
Programmable valves and actuators are widely used in man-made systems to provide sophisticated control of fluid flows. In nature, however, this process is frequently achieved using passive soft materials. Here we study how elastic deformations of cylindrical pores embedded in a flexible membrane enable passive flow control. We develop biomimetic valves with variable pore radius, membrane radius, and thickness. Our experiments reveal a mechanism where small deformations bend the membrane and constrict the pore - thus reducing flow-while larger deformations stretch the membrane, expand the pore, and enhance flow. We develop a theory capturing this highly nonmonotonic behavior, and validate the scaling across a broad range of material and geometric parameters. Our results suggest that intercompartmental flow control in living systems can be encoded entirely in the physical attributes of soft materials. Moreover, this design could enable autonomous flow control in man-made systems.
\end{abstract}

DOI: 10.1103/PhysRevLett.125.098101

Introduction.-Many industries rely on precise, automated flow control: from chemical processing to dialysis machines and implants [1-4]. A host of valve designs and controllers can provide this flow regulation, but the expense of these systems often limits the scope of their application [5-7]. In nature, similarly refined flow control is essential. Compartmentalization is a hallmark of multicellular life, and distinct biological functions and physiochemical processes tend to occur in physically separate domains. Organisms are able to control the transport of fluids between these compartments at many scales: from cells and subcellular organelles to tissues and organs. The pores are often embedded in a soft matrix which can deform due to stress [8-10]. However, the link between elastic deformations and flow remains unclear.

Channels connecting distinct compartments in fungi, plants, and animals play an essential role across many scales: for instance in growth and tissue patterning, nutrient and energy distribution, and defense against pathogens. In fungi, the continuous cytoplasm of the mycelia is compartmentalized by soft perforated septa [Fig. 1(a)]. These allow streaming of cytoplasm and translocation of organelles like mitochondria and nuclei [11-13]. Under extreme environmental conditions, septa can close [8] to prevent loss of cytoplasm [14-16] and subsequent death. Similar structures are ubiquitous in plants, where plasmodesmata nanopores $[10,17]$, micron-scale phloem sieve pores [18], and xylem pit pores [19-22] allow plants to control growth, enable cellcell trafficking of water, limit pathogen movement, and prevent catastrophic water loss [Fig. 1(a)]. Indeed, several of these transport processes have been shown to exhibit a strong pressure dependence [23] or solute concentration threshold for transport [24], a hallmark of soft matter interactions. In animals, tissue-scale valves provide complex nonlinear flow control, sometimes maintaining directionality to the flow. In insects, primitive heart valves (ostia) provide nonlinear flow control very similar to cellular pores found in fungi and plants [25]. In mammals, similar pressure-sensitive millimeter-scale valves are present in veins and the heart.

Remarkably, these valves share a common features that have evolved across biological kingdoms and sizes: an open cylidrical pore embedded in an impermeable soft matrix. Structural changes in the matrix - in response to mechanical, chemical, or thermal stress [28-30]— cause the pore shape to change, thus impacting flow across individual pores (Fig. 1) and in channel networks [31]. Deformations of perforated rigid plates $[32,33]$ or bending of elastic plates $[34,35]$ have been widely investigated. However, to date, few experiments have addressed the processes that link elastic deformations and fluid flows in this geometry [36].

Accordingly, in this report we investigate the interplay of hydrodynamics and permeability of soft pores [Fig. 1(c)]. The objective of this Letter is to study the deformation response of this poroelastic process and uncover how passive feedback properties are encoded in simple elastic and geometric features. As we demonstrate below, cylindrical pores in soft sheets can act as passive valves. Experimental data match our predictions for both the pore deformations and for the hydraulic resistance across five orders of magnitude in pressure (see Figs. 3 and 4). In a broader biomimetic perspective, our findings point to new applications in microfluidics, morphing skins and soft actuators or robots [37-40].

Results.-The system under consideration is an idealized model of a biological channel: a cylindrical pore in a circular elastic sheet separating two compartments [Figs. 1(b), 1(c), 
(a) nano- and micro pores

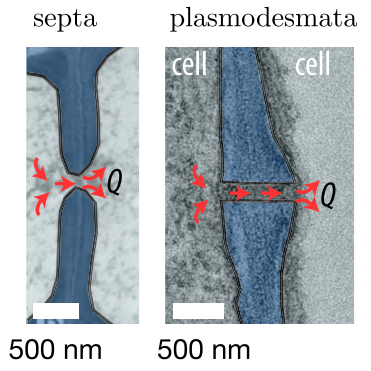

(b) opening mechanism

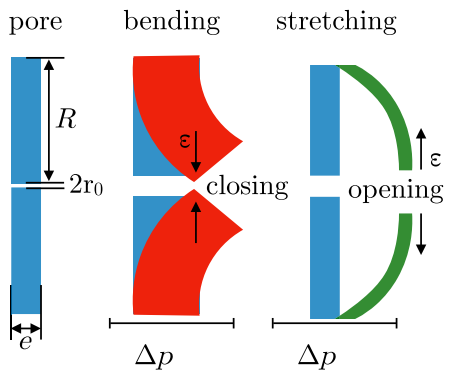

(c) passive flow response

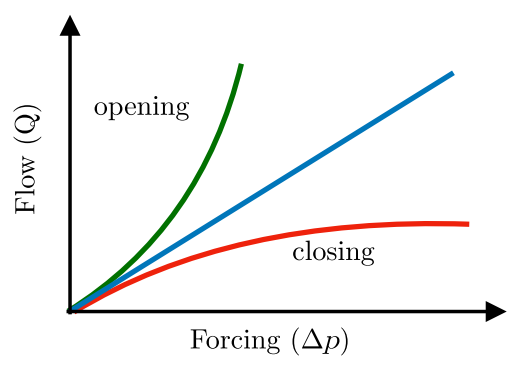

FIG. 1. Controlling flow through soft intercompartmental channels. (a) In many living tissues flow is controlled by soft valves that regulate essential processes from morphogenesis to disease response. Examples include septal nanopores in fungi and plasmodesmata in plants. (b) We propose that elastic processes enable the pore to either close (when bending dominates) or open (when stretching dominates) due to strain $\varepsilon$ in the surrounding tissue induced by the application of a pressure drop $\Delta p$ across the pore. (c) In several systems, the transport flow rate $Q$ between two compartments depends nonlinearly on the forcing (pressure difference $\Delta p$ ) due to the mechanical properties of soft materials in the pores. Panel (a) adapted from [26] and [27] reproduced with permission.

and 2(a)]. To elucidate the relationship between mechanical stress, pore deformation, and permeability, experiments on pressure-driven flow were carried out as described in the Supplemental Material [41]. Briefly, we measured the hydraulic resistance $R_{\text {hyd }}=\Delta p / Q$, which is the ratio of the applied pressure difference $\Delta p$ and the fluid flow rate $Q$ across the pore. These experiments, and direct measurements of the pore radius $r$ as function of pressure, were carried out for a range of system parameters (see details in the Supplemental Material [41]).

The hydraulic properties of the system are illustrated in Figs. 2(b) and 2(c). At low pressures, flow increases approximately linearly with pressure corresponding to a constant hydraulic resistance $R_{\text {hyd }}^{(0)}=\Delta p / Q$ of a stiff plate in the undeformed state [Figs. 2(b) and 2(c)]. However, as pressure increases, we observe two distinct behaviors: for membranes that undergo modest deformations and bend, the hydraulic resistance $R_{\text {hyd }}$ grows with pressure. In contrast, the opposite behavior is observed for membranes that experience large deformations and stretch: the hydraulic resistance decays with pressure. The observed variation in flow resistance is consistent with direct measurements of pore size $r$ as a function of applied pressure: pore size decreases in the bending regime and increases as the membrane is stretched [Fig. 3(a)]. We can observe an intermediate regime in between the bending and stretching regimes, where the hole reaches a minimum aperture corresponding to the energy balance between bending and stretching. In addition, we also observed that the magnitude of the pore deformation depends on the membrane dimension; specifically, it varies significantly with the radius $R$ and thickness $e$ of the elastic plate.

To rationalize the experimental data, we first consider the relationship between flow rate $Q$ and applied pressure $\Delta p$ (Fig. 2). While the full problem presented in Fig. 1(c) could be solved for individual cases using, for instance, a finite-element package [43-45], we choose to use scaling methods that captures the different physical regimes of our experiments and allow us to extract scalings of the relevant parameters.

To elucidate the variation of pore size $r$ with applied pressure $\Delta p$, we consider the elastic deformation of the
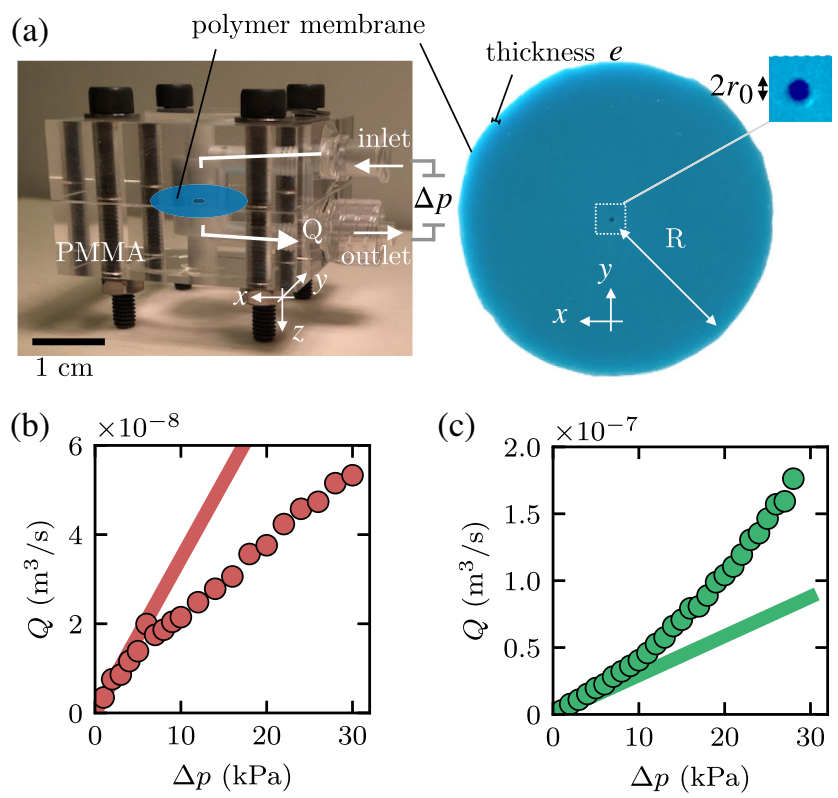

FIG. 2. Experimental setup and results. (a) Photograph of the experimental setup. A perforated polymer membrane of radius $R$ and thickness $e$ is clamped between two pieces of PMMA with an embedded channel. The pressure drop $\Delta p$ drives the flow rate $Q$ across the small pore of equilibrium radius $r_{0}$. (b) Measured flow rate as a function of applied pressure $\Delta p$ for a plate of radius $R=5 \mathrm{~mm}$. The flow rate increases sublinearly with pressure for relatively thick membranes (red dots), while it (c) increases superlinearly with pressure for relatively thin membranes (green dots). Note that, for rigid plates, the flow rate and pressure vary linearly (straight lines). The experimental parameters are listed in Table I, see [41]. 

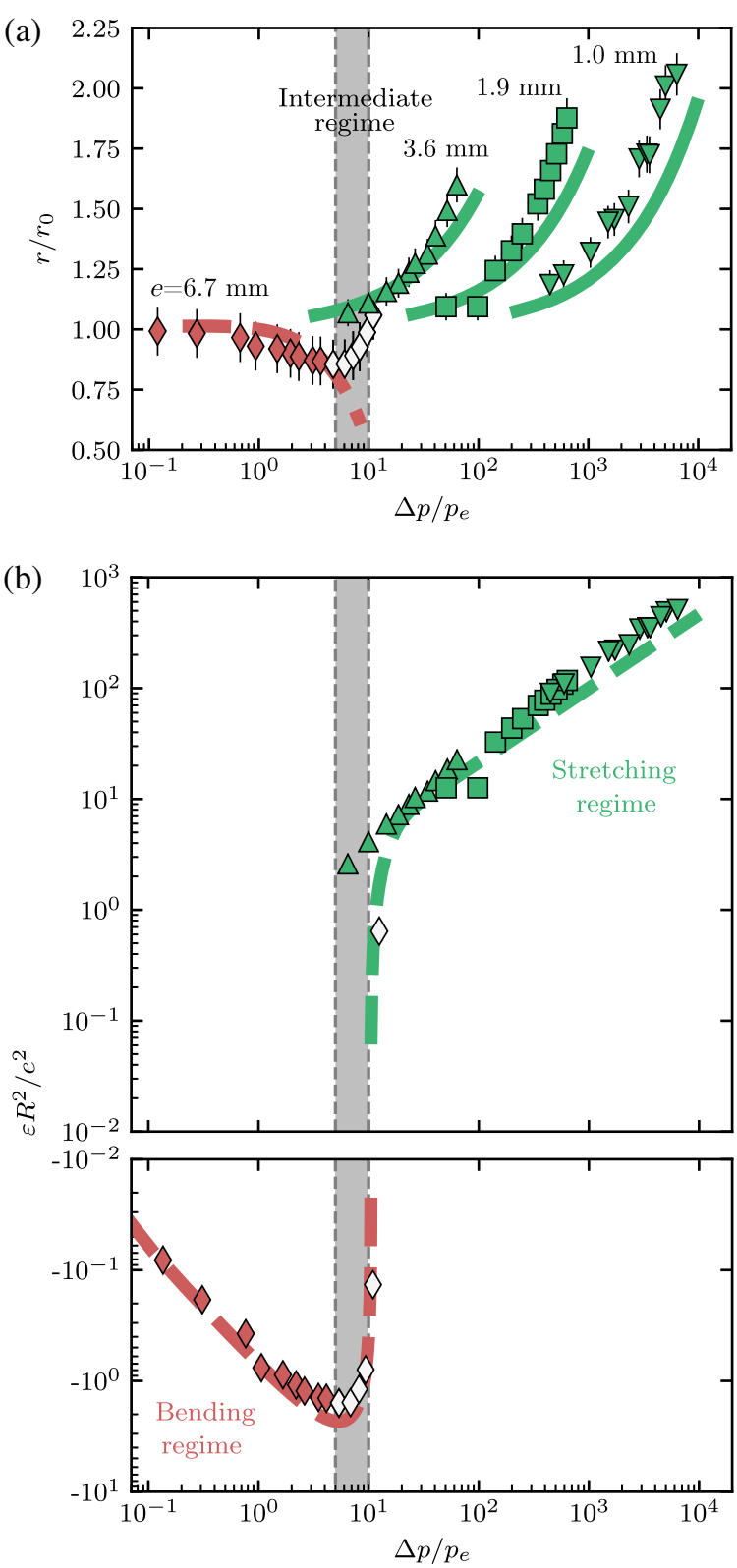

FIG. 3. Deformation strongly impacts pore radius. (a) Measured relative pore radius $r / r_{0}$ plotted as a function of applied pressure $\Delta p / p_{e}$ for a $r_{0}=130 \mu \mathrm{m}$ pore in a $R=22 \mathrm{~mm}$ soft plate of thickness $e$ as indicated in the figure. The green solid lines indicate theoretical results in the stretching limit [Eq. (2b)] with $\beta=1$, while the red dashed line is the sum of stretching and bending contributions $\varepsilon_{b}+\varepsilon_{s}$ [Eqs. (2b) and (2a)] with $\alpha=\beta=1$. Note the bending-to-stretching transition around $\Delta p / p_{e}=5-10$ in the $e=$ $6.7 \mathrm{~mm}$ experiment [42]. (b) Normalized pore strains $\varepsilon\left(R^{2} / e^{2}\right)=$ $R^{2}\left(r-r_{0}\right) /\left(r_{0} e^{2}\right)$ as a function of applied pressure $\Delta p / p_{e}$. In the bending regime (red diamonds), the strain scales linearly with the pressure $\Delta p / p_{e}$, as predicted by our theory [Eq. (2a)]. As pressure increases across the intermediate regime $\Delta p / p_{e}=5-10$ (shaded region), we recover the stretching limit, where strain scales as $\left(\Delta p / p_{e}\right)^{2 / 3}$ in accord with Eq. (2b). The dashed line represents a sigmoidal superposition of the two models with $\alpha=\beta=1$ (see the Supplemental Material [41]). The experimental parameters are listed in Table I, see [41]. membrane, which follows well-established plate theory [46]. Thin elastic plates are characterized by a linear relationship between the applied load and the maximum deflection amplitude $\chi \sim \Delta p R^{4} /\left(E e^{3}\right)$, where $E$ is Young's modulus [47]. This formula is valid at low pressures when the deformations are bending dominated and remain small relative to the plate thickness $e$ [Fig. 1(b)] [46]. This corresponds to the limit where the applied load $\Delta p$ is less than the characteristic elastic pressure

$$
p_{e}=E \frac{e^{4}}{R^{4}}
$$

In the bending-dominated case we can thus express the deformation by the formula

$$
\frac{\chi}{e} \sim \frac{\Delta p}{p_{e}} \text { for } \Delta p<p_{e} .
$$

When the applied pressure exceeds the small-deformation limit $\left(\Delta p>p_{e}\right)$, stretching dominates and the deflection amplitude scales with the $1 / 3$ power of pressure [48]

$$
\frac{\chi}{e} \sim\left(\frac{\Delta p}{p_{e}}\right)^{1 / 3} \text { for } \Delta p>p_{e} .
$$

The transition between the two regimes [Eqs. (1b) and (1c)] occurs in the relative pressure range $\Delta p / p_{e}=5-10$, see [42].

To account for our experimental observations (Fig. 2) we consider the variation in pore radius $r$ generated by the membrane deformations [Eqs. (1b)-(1c)], because a smaller pore permits less flow. The relative change in the pore aperture $\left(r-r_{0}\right) / r_{0} \sim \varepsilon$ is proportional to the membrane strain $\varepsilon$ which can be induced by either bending or stretching [46]. Here, $r_{0}$ is the radius of the undeformed pore, and the dynamic pore radius is $r=r_{0}(1+\varepsilon)$.

The strain in the bending-dominated case is given by $\varepsilon \sim-e / R_{n}$, where $R_{n}$ is the radius of curvature $R_{n}=$ $R^{2} /(2 \chi)$ of the deformed state. This leads to

$$
\varepsilon_{b}=-\alpha\left(\frac{e}{R}\right)^{2} \frac{\Delta p}{p_{e}},
$$

where $\alpha$ is an $\mathcal{O}(1)$ numerical constant, dependent on the detailed domain shape and boundary conditions. We observe that the pore-radius theory [Eq. (2a)] is in reasonable agreement with experimental observations for $\alpha=1$ (Fig. 3).

Proceeding to consider the stretching-dominated case, we once again consider the pore strain $\varepsilon$. A membrane subject to large deflections is under tension, and the strain is given by $\varepsilon_{s} \sim T /(E e)$, where $T \sim R_{n} \Delta p$ is the membrane tension. With Eq. (1c), this leads to the relative change in pore aperture 


$$
\varepsilon_{s}=\beta\left(\frac{e}{R}\right)^{2}\left(\frac{\Delta p}{p_{e}}\right)^{2 / 3}
$$

where $\beta$ is an $\sim \mathcal{O}(1)$ numerical constant. The pore-radius theory [Eq. (2b)] is in good agreement with experimental observations with $\beta=1$ (Fig. 3).

To elucidate the impact of the pore constriction [Eq. (2a)] and expansion [Eq. (2b)] on the flow characteristics (Fig. 2), we consider the hydrodynamic properties of the membrane. Assuming a low-Reynolds-number flow in the pore, the flow-rate vs pressure-drop relation is given by

$$
\frac{\Delta p}{Q}-R_{\mathrm{sys}}=R_{\mathrm{hyd}}=\frac{8 \eta e}{r^{4}},
$$

where $R_{\text {sys }}$ is the (constant) resistance of the inlet and outlet tubing in the experimental setup. $R_{\text {hyd }}$ is the flow resistance in the pore which scales linearly with the liquid viscosity $\eta$ and plate thickness $e$ and inversely with the fourth power of the pore radius $r$, according to the Hagen-Poiseuille law. A change in pore aperture size induced by elastic deformations is thus expected to strongly impact flow [49], in qualitative agreement with our experimental observations [Fig. 3(b)].

Applying the pressure-strain relations (Fig. 3) to the pressure-drop vs flow-rate relation in Eq. (3a) leads to the relative hydraulic resistance

$$
\frac{R_{\mathrm{hyd}}}{R_{\mathrm{hyd}}^{(0)}}=\frac{r_{0}^{4}}{r^{4}}=(1+\varepsilon)^{-4},
$$

where $R_{\text {hyd }}^{(0)}=8 \eta e / r_{0}^{4}$ is the hydraulic resistance of the undeformed pore. It is apparent that strain induces strong variations in the flow resistance. In the bending limit $\left(\Delta p<p_{e}\right)$, where $\varepsilon<0$ [Eq. (2a)] the resistance increases with pressure, in reasonable accord with data (Fig. 4). In the stretching limit $\left(\Delta p>p_{e}\right)$, where $\varepsilon>0$ [Eq. (2b)], the hydraulic resistance decreases consistent with experimental observations (Fig. 4).

Our analysis has focused on the limiting behavior where either bending or stretching dominates. In general however, both effects contribute and a pore initially blocked by bending (when $\varepsilon_{b}=-1$ ) could reopen at a higher pressure where stretching $\varepsilon_{s}>1$ as shown in Figs. 3(a) and 4. This is consistent with our observations; however, some experiments showed signs of hysteresis. Indeed, the transition regime cannot necessarily be explained in detail by our model since the closed pore may be locked into a state that requires a higher pressure difference to unjam.

Discussion and conclusion.-A fairly comprehensive picture of the elements that influence viscous pressuredriven flow across a soft perforated membrane has come into view. Most prominent is the experimental discovery that this system can act as a simple nonlinear hydraulic

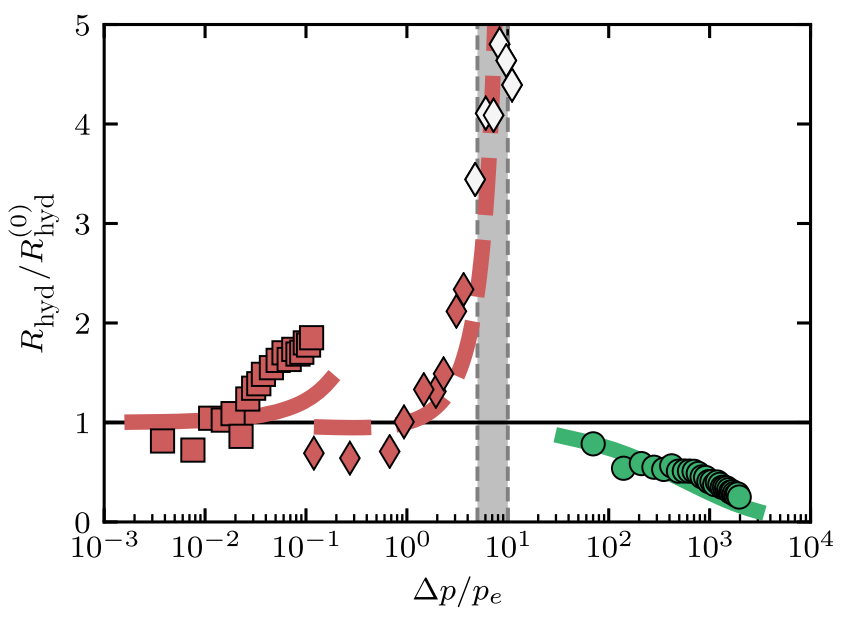

FIG. 4. Normalized hydraulic resistance $R_{\text {hyd }} / R_{\text {hyd }}^{(0)}$ plotted as function of normalized pressure $\Delta p / p_{e}$. The lines indicate theoretical predictions [Eq. (3b)] based on strain $\varepsilon$ in the stretching limit [Eq. (2b), green solid line], the transition regime [the sum of Eqs. (2b) and (2a), red dashed line], and the bending regime [Eq. (2a), red solid line]. Note the abruptly decreasing hydraulic resistance near the end of the transition regime (shaded region). The experimental parameters are listed in Table I.

resistor, with dramatic impact on flow. The quantitative behavior of the device depends on the material and geometric parameters of the system [Eqs. (2a) and (2b)]. The valve characteristics are completely determined by the membrane thickness-to-radius ratio $e / R$ and the characteristic elastic pressure $p_{e}$ [Eq. (1a)], which marks the transition from bending to stretching (Figs. 3 and 4). When a moderate pressure is applied, flow increases sublinearly with pressure as bending of the membrane causes a reduction in the pore aperture. This trend is reversed at higher pressures, where the stretching of the membrane dominates and flow increases super-linearly with pore size. The transition between these two regimes occurs when the elastic deformation is comparable to the membrane thickness. We observe good agreement between experimental data and simple theoretical predictions.

The structures under consideration here are frequently observed in nature (Fig. 1). However, while the effects of fluid-structure interactions have been widely studied, e.g., in blood vessels [50], the implications of elastic deformations for biological function of pore apertures embedded in soft structures are not yet fully understood. To our knowledge, this study represents the first step in elucidating the effects large deformations of poroelastic membranes have on intercompartment transport. One example is plasmodesmata pores that provide a continuous liquid bridge between neighboring plant cells (Fig. 1, [17]). The flow-control mechanisms described here may play a role in mitigating the effects of tissue damage and osmotic shock, which can induce substantial cell-to-cell pressure differences. The increased plasmodesmatal hydraulic resistance induced by bending of the cell wall [Eq. (3b)] could delay the 
pressure-driven loss of cytoplasmic liquid, and may allow time for permanent blockage of the pores by callose deposition [23]. In performing this function, it could complement pressure-induced movement of the ER complex [17]. Similarly, the squeezing of nuclei and mitochondria through fungal septa pores [Fig. 1(a)] could be facilitated by a pressure-induced increase in the pore aperture [51]. We note that transport across plant cell walls occurs across many plasmodesmata in parallel. However, since the pores are relatively dilute and far apart, pore-to-pore interactions are negligible (for effects of pore density, see, e.g., [52,53]). Finally, our results also have potential implications for the pit membranes that link adjacent conductive elements in the xylem of vascular plants [54]. These porous screens prevent air from moving between cells, but offer significant resistance to intercellular water flow. Our results support a mechanism for reducing the impact of the pit filters: membrane stretching could decrease the overall hydraulic resistance of the water transport pathway or prevent gas bubble propagation by reducing the pore aperture [55].

The system size in our experiments is larger than in most of the aforementioned biological cases. However, flow and deformation are governed by the same basic physical laws at the scale of cell-cell pores, i.e., viscosity-dominated hydrodynamics and elastic membrane deformations. To compute the impact of pressure on the hydraulic resistance at cellular scales using Eq. (3b), it is necessary to evaluate the pore strain $\varepsilon=\left(r-r_{0}\right) / r_{0}$ using Eqs. (2a) and (2b). Inspection of these expressions reveals two relevant nondimensional quantities: the membrane thickness-to-radius ratio $e / R$ and the ratio of applied-to-characteristic pressure, $\Delta p / p_{e}$. To translate between our macroscopic experiments and living cells, we must therefore ensure that these quantities are of similar magnitude in the two systems. Our experiments cover the range $e / R \approx 0.05-0.5$ and $\Delta p / p_{e} \approx 10^{-1}-10^{4}$, corresponding to the full transition between bending and stretching-dominated regimes in moderately thin plants. Additional, the pore length-todiameter aspect ratio was $e /\left(2 r_{0}\right) \approx 5-50$ in our system. The absolute size of pores in, e.g., plants and fungi are somewhat smaller, with typical dimensions in the range $e \approx 0.5-10 \mu \mathrm{m}, \quad R \approx 1-100 \mu \mathrm{m}, \quad$ and $\quad r_{0} \approx 0.05-1 \mu \mathrm{m}$. However, the typical geometric ratios $e / R \approx 0.01-0.5$ and $e /\left(2 r_{0}\right) \approx 5-10$ overlap with our experiments. Similarly, the pressure ratio in, for instance, fungal septa, varies between $\Delta p / p_{e} \sim 1-10^{3}[26,56,57]$. Hence, we anticipate our conclusions to translate directly to cellular scales. We note, however, that direct in vivo experiments are required to validate the expected pressure-induced pore strains. Such experiments are challenging due to the size of the pores under consideration in plants and fungi, and the difference in the plane of observation to directly visualize the membrane deflection and the pore strain. It may, however, be possible to use 3D reconstructions (based on, e.g., confocal microscopy) to overcome this difficulty.
In conclusion, we emphasize that flow control is also highly relevant to man-made systems, and valve design in microdevices remains a major challenge for many applications, e.g., drug delivery, fuel dispensing, soft robotics, and surgery [58-61]. In this study, we reveal a generic mechanism for flow control using fluid-structure interactions in a perforated membrane, a step toward the design and fabrication of entirely soft, complex, autonomous flow control systems.

This work was supported by a research grant (Grant No. 17587) from VILLUM FONDEN. We thank B. B. Browne for valuable insights and feedback.

*khjensen@fysik.dtu.dk

[1] S. R. Ash, Continuous flow-through peritoneal dialysis (cfpd) method with control of intraperitoneal pressure, U.S. Patent No. 6,409,699 (2002).

[2] R. Spohr, N. Reber, A. Wolf, G. M. Alder, V. Ang, C. L. Bashford, C. A. Pasternak, H. Omichi, and M. Yoshida, J. Controlled Release 50, 1 (1998).

[3] O. Nissan, S. Ben-Rimon, and I. Yaron, Delivery devices for flow regulating implants, U.S. Patent App. 09/987,134 (2003).

[4] Z. Zhang, D. Jones, S. Yue, P. Lee, J. Jones, C. Sutcliffe, and E. Jones, Mater. Sci. Eng. 33, 4055 (2013).

[5] A. W. Harrison, Hydraulic flow control valves, U.S. Patent No. 4,508,140 (1985).

[6] C. Yu, S. Mutlu, P. Selvaganapathy, C. H. Mastrangelo, F. Svec, and J. M. Fréchet, Anal. Chem. 75, 1958 (2003).

[7] E. Bazigou and T. Makinen, Cell Mol. Life Sci. 70, 1055 (2013).

[8] A. F. Van Peer, W. H. Müller, T. Boekhout, L. G. Lugones, and H. A. Wösten, PLoS One 4, e5977 (2009).

[9] M. Capron, P. Tordjeman, F. Charru, E. Badel, and H. Cochard, Phys. Rev. E 89, 033019 (2014).

[10] K. Park, J. Knoblauch, K. Oparka, and K. H. Jensen, Nat. Commun. 10, 1 (2019).

[11] R. Bauer, D. Begerow, J. P. Sampaio, and M. Weiß, and F. Oberwinkler, Mycol. Prog. 5, 41 (2006).

[12] D. Barr, S. Huhndorf, D. McLaughlin, E. McLaughlin, and P. Lemke, The Mycota VII Part A. Systematics and Evolution, edited by D. J. McLaughlin, E. G. McLaughlin, and P. A. Lemke (Springer, Berlin, 2001), pp. 93-112.

[13] J. Fell, T. Boekhout, A. Fonseca, and J. Sampaio, The Mycota VII. Systematics and Evolution. Part b, edited by D. J. Mclaughlin, E. G. Mclaughlin, and P. A. Lemke (Springer-Verlag, Berlin, 2001), pp. 85-120

[14] R. Aylmore, G. Wakley, and N. Todd, Microbiology 130, 2975 (1984).

[15] P. Markham, Mycol. Res. 98, 1089 (1994).

[16] A. Trinci and A. J. Collinge, Protoplasma 80, 57 (1974).

[17] W. J. Nicolas, M. S. Grison, S. Trépout, A. Gaston, M. Fouché, F. P. Cordelières, K. Oparka, J. Tilsner, L. Brocard, and E. M. Bayer, Nat. Plants 3, 17082 (2017).

[18] J. Knoblauch, S. Tepler Drobnitch, W. S. Peters, and M. Knoblauch, Plant, Cell Environ. 39, 1727 (2016). 
[19] B. Choat, S. Jansen, M. A. Zwieniecki, E. Smets, and N. M. Holbrook, J. Exp. Bot. 55, 1569 (2004).

[20] J. S. Sperry and U. G. Hacke, Am. J. Bot. 91, 369 (2004).

[21] B. Choat, A. R. Cobb, and S. Jansen, New Phytol. 177, 608 (2008).

[22] S. Delzon, C. Douthe, A. Sala, and H. Cochard, Plant, Cell Environ. 33, 2101 (2010).

[23] K. Oparka and D. Prior, Plant J. 2, 741 (1992).

[24] M. A. Zwieniecki, P. J. Melcher, and N. M. Holbrook, Science 291, 1059 (2001).

[25] L. T. Wasserthal, J. Exp. Bot. 210, 3707 (2007).

[26] T. Boekhout, Y. Yamada, A. Weijman, H. Roeymans, and W. Batenburg-Van der Vegte, Syst. Appl. Microbiol. 15, 1 (1992).

[27] T. J. Ross-Elliott et al., Elife 6, e24125 (2017).

[28] R. Aloni, A. Raviv, and C. Peterson, Can. J. Bot. 69, 1825 (1991).

[29] K. van Driel, A. van Peer, W. Muller, T. Boekhout, and H. Wosten, Dissertation, University of Utrecht, 2007.

[30] D. Mullendore, C. Windt, H. Van AS, and M. Knoblauch, Plant Cell 22, 579 (2010).

[31] M. Ruiz-Garcia and E. Katifori, arXiv:2001.01811.

[32] C. Lo and A. Leissa, Acta Mech. 4, 64 (1967).

[33] C. Atanasiu and St. Sorohan, Mater. Sci. Eng. 147, 012095 (2016).

[34] C. van Rijin, M. van der Wekken, W. Nijdam, and M. Elwenspoek, J. Microelectromech. Syst. 6, 48 (1997).

[35] T. Liu, A. Jagota, and C. Hui, Soft Matter 13, 386 (2017).

[36] A. H. Christensen and K. H. Jensen, Phys. Rev. Fluids 5, 044101 (2020).

[37] C. Thill, J. Etches, I. Bond, K. Potter, and P. Weaver, Aeronaut. J. (1968) 112, 117 (2008).

[38] N. Lu and D.-H. Kim, Soft Rob. 1, 53 (2014).

[39] J. Shintake, B. Schubert, S. Rosset, H. Shea, and D. Floreano, in 2015 IEEE/RSJ International Conference on Intelligent Robots and Systems (IROS) (IEEE, New York, 2015), pp. 1097-1102.

[40] D. Vella, Nat. Rev. Phys. 1, 425 (2019).

[41] See the Supplemental Material at http://link.aps.org/ supplemental/10.1103/PhysRevLett.125.098101 for sections contained in the supplemental file: Membrane fabrication,
Flow setup, Pore size setup, Sigmoidal interpolation between pressure regimes, and Experimental parameters.

[42] Y. Bouremel, S. Madaan, R. M. Lee, I. Eames, A. Wojcik, and P. T. Khaw, J. Fluids Struct. 70, 261 (2017).

[43] C. Duprat and H. A. Stone, Fluid-Structure Interactions in Low-Reynolds-Number Flows (Royal Society of Chemistry, Cambridge, 2015).

[44] E. H. Dowell and K. C. Hall, Annu. Rev. Fluid Mech. 33, 445 (2001).

[45] G. Hou, J. Wang, and A. Layton, Commun. Comput. Phys. 12, 337 (2012).

[46] S. Timoshenko and J. Goodier, Theory of elasticity (McGraw-Hill, New York, 1969).

[47] C. Coulais, J. T. B. Overvelde, L. A. Lubbers, K. Bertoldi, and M. van Hecke, Phys. Rev. Lett. 115, 044301 (2015).

[48] R. Jones, Int. J. Nonlinear Mech. 9, 141 (1974).

[49] H. Bruus, Theoretical Microfluidics (Oxford University Press, New York, 2008).

[50] M. Heil and A. L. Hazel, Annu. Rev. Fluid Mech. 43, 141 (2011).

[51] D. Hunsley and G. Gooday, Protoplasma 82, 125 (1974).

[52] B. Florijn, C. Coulais, and M. van Hecke, Soft Matter 12, 8736 (2016).

[53] D. Chen, Compos. Struct. 93, 736 (2011).

[54] J. Pittermann, B. Choat, S. Jansen, S. A. Stuart, L. Lynn, and T. E. Dawson, Plant Physiol. 153, 1919 (2010).

[55] A. Tixier, S. Herbette, S. Jansen, M. Capron, P. Tordjeman, H. Cochard, and E. Badel, Ann. Bot. 114, 325 (2014).

[56] R. R. Lew, Nat. Rev. Microbiol. 9, 509 (2011).

[57] M. Haneef, L. Ceseracciu, C. Canale, I. S. Bayer, J. A. Heredia-Guerrero, and A. Athanassiou, Sci. Rep. 7, 1 (2017).

[58] D. P. Holmes, B. Tavakol, G. Froehlicher, and H. A. Stone, Soft Matter 9, 7049 (2013).

[59] J. B. Overvelde, T. Kloek, J. A. D'haen, and K. Bertoldi, Proc. Natl. Acad. Sci. U.S.A. 112, 10863 (2015).

[60] M. Gomez, D. E. Moulton, and D. Vella, Phys. Rev. Lett. 119, 144502 (2017).

[61] P. Rothemund, A. Ainla, L. Belding, D. J. Preston, S. Kurihara, Z. Suo, and G. M. Whitesides, Sci. Rob. 3, eaar7986 (2018). 